Www.jmscr.igmpublication.org

Impact Factor (SJIF): 6.379

Index Copernicus Value: 79.54

ISSN (e)-2347-176x ISSN (p) 2455-0450

crossrefDOI: https://dx.doi.org/10.18535/jmscr/v6i8.159

Journal Of Medical Science And Clinical Research

IGM Publication

An Official Publication of IGM Publication

\title{
Role of modified WHO partograph in the management of labour in nulliparous women-a prospective study
}

\author{
Authors \\ Dr Pankhuri Goyal Busar ${ }^{1}$, Dr Sonam Chaudhary ${ }^{2}$, Dr Kavita Sharma ${ }^{3}$ \\ Dr Seema Sharma ${ }^{4}$ \\ ${ }^{1}$ Assistant Professor, ${ }^{2}$ III ${ }^{\text {rd }}$ Year Resident, ${ }^{3}$ Senior Resident, ${ }^{4}$ Professor,
}

Dept. of Obstetric \& Gynecology, Mahatma Gandhi Medical College \& Hospital, Jaipur, Rajasthan, India

*Corresponding Author

Dr Sonam Chaudhary

III $^{\text {rd }}$ Year Resident, Dept. of Obstetric \& Gynecology, Mahatma Gandhi Medical College \& Hospital, Jaipur,

Rajasthan, India

\section{Abstract}

Background: Partograph is a single standardized sheet on which midwives, nurses and doctors can record their examination findings to generating of pictorial overview of labour progress and maternal and fetal condition. We aim to assess the role of modified WHO partograph in management of labour in nulliparous patients with spontaneous onset of labour pain at a tertiary care centre.

Material \& Methods: This prospective study was done in the labour room of Obstetric and Gynecology department of Mahatma Gandhi Medical College and Hospital, Jaipur, Rajasthan from March 2018 to April 2018. 140 nulligravida women fulfilling the inclusion and exclusion criteria were included after written informed consent. Patient details, progression of labour after $4 \mathrm{~cm}$ of cervical dilatation and monitoring of maternal and fetal condition was recorded on the WHO modified partograph in all patients.

Results: Mean age of the patient was 23.13 years, mean BMI was $22.77 \mathrm{~kg} / \mathrm{m} 2$ and majority belonged to urban society. Mean gestation age at presentation in labour room was 39.3 weeks. The majority 118 $(84.28 \%)$ delivered vaginally, $6(4.28 \%)$ by assisted vaccum delivery and $22(15.71 \%)$ needed emergency LSCS. Maternal complications (3.57\%) namely atonic PPH (2), extended episiotomy (2) and precipitate labour with complete perineal tear (1) were seen in cases of abnormal progress of labour, all managed properly. There was no neonatal \& maternal mortality.

Conclusion: We suggested that in a country like India, where still maternal morbidity and mortality rate is high, partograph use should be implemented in all hospitals and labour rooms for better obstetric care and attainment of Millennium Development Goal of improving maternal health.

Keywords: Partograph, Neonatal outcome, Mode of Delivery, Maternal outcome.

\section{Introduction}

"Partograph" is a greek word meaning "labour or parturition curve". It is a graphical representation of a women's progress of labour along with relevant details of the women and fetus. It was first described by Friedmann ${ }^{1}$ in 1954 as a ' $\mathrm{S}$ ' shaped sigmoid curve and later modified by Philpott and Castle ${ }^{2}$ in 1972. 
Partograph is a single standardized sheet on which midwives, nurses and doctors can record their examination findings, generating a pictorial overview of labour progress and maternal and fetal condition. It serves as an early warning system, assisting in early diagnosis of abnormal labour, timely decision on referral, augmentation ,and if necessary, termination of labour by caesarean section.

After its critical role in preventing both maternal and perinatal morbidity and mortality was recognized, especially in developing countries, WHO accepted it in $1987^{3}$. But, later modifications were made in original composite partograph. Latent phase was removed and definition of active phase was changed to commence at $4 \mathrm{~cm}$ of cervical dilation instead of $3 \mathrm{~cm}$ used previously. Alert and action line were kept as such at an interval of 4 hours, crossing which will point towards abnormal progress of labour.

Maternal mortality rate (MMR) in India (2015) was 174 (139-217) per 1 lakh live births and neonatal mortality rate was about 26.4(23.9-29.2) per 1000 birth. In Rajasthan ${ }^{4}$, where this study is done, has MMR of 225 much higher than of Kerala having MMR of only $66^{5}$

India still accounts for $1 / 5^{\text {th }}$ of global burden of maternal deaths $(17 \%)$. It is estimated that for each death of a mother, there are 15 who escaped near death by chance or are alive but debilitated. Post partum haemorrhage (33\%), antepartum haemorrhage $(7.1 \%)$, sepsis $(11.1 \%)$, pregnancy induced hypertension (16.1\%), obstructed labour $(5 \%)$ etc., account for major causes of maternal mortality and morbidity. ${ }^{6}$ Most common underlying cause of these is 'delay', in recognition or referral or intervention of abnormal and prolonged labour. These can be prevented to a large extent by use of simplified modified partograph. In this study, we aim to assess the role of modified WHO partograph in management of labour in nulliparous patients with spontaneous onset of labour pain at a tertiary care centre.

\section{Methodology}

This prospective study was done in the labour room of Obstetric and Gynecology department of Mahatma Gandhi Medical College and Hospital, Jaipur, Rajasthan from March 2018 to April 2018. 140 nulliparous women fulfilling the inclusion and exclusion criteria were included after written informed consent.

\section{Inclusion Criteria}

-Nullipara

-singleton pregnancy

-after 37 completed weeks

-cephalic presentation

-spontaneous onset of labour

\section{Exclusion Criteria}

-severe pregnancy induced hypertension

-severe anemia

-uncontrolled Diebetes Mellitus

-major degree of Cephalo Pelvic Disproportion

-malpresentation

-antepartum hemorrhage

-multiple pregnancy

-Intra Uterine fetal death

After written informed consent, detailed history regarding age, parity, duration of pregnancy and labour pains was taken. General physical examination and abdominal examination was done. Fundal height, lie, presentation, engagement, uterine contractions and fetal heart rate noted. Per vaginal examination was done for cervical dilatation, effacement, state of membranes, liquor and pelvic assessment. All the data were noted in the proforma.

Patient details, progression of labour after $4 \mathrm{~cm}$ cervical dilatation and monitoring of maternal and fetal condition was recorded on the WHO modified partograph in all patients.

Each partograph was analysed for its role in influencing decision making in labour management.

Progress of labour curve, shift beyond alert and action line, duration of labour, need for augmentation or intervention, mode of delivery - 
vaginal/ assisted vaginal/ LSCS was noted and perinatal outcome studied by APGAR score at birth and NICU admission recorded. Statistical analysis was done by using the results.

\section{Results}

Total 140 nulliparous (fulfilling the inclusion and exclusion criteria) patients in labour were monitored with WHO modified partograph (figure 1). Mean age of the patient was 23.13 years, mean BMI was $22.77 \mathrm{~kg} / \mathrm{m} 2$ and majority belonged to urban society. Mean gestation age at presentation in labour room was 39.3 weeks (table 1).

As shown in table 2, majority $118(84.28 \%)$ delivered vaginally including $6(4.28 \%)$ by assisted vaccum delivery and $22(15.71 \%)$ needed emergency LSCS (table 2).

Of all patients, $80(57.14 \%)$ who remained to left of alert line delivered vaginally. For patients with curve falling in between alert and action line, proper reassessment was done and augmentation of labour for slow descent and dilatation was done which resulted in another $32(22.85 \%)$ of vaginal delivery.

Vaccum was applied in 6 patients $(4.28 \%)$ for indications like prolonged $2^{\text {nd }}$ stage, inability to bear down properly and fetal distress in $2^{\text {nd }}$ stage of labour.

Among 22 LSCS (15.71\%), various indications were fetal distress and meconium stained liquor in $7(31.81 \%)$ patients each, non progress of labour in $9(40.90 \%)$ patients and deep transverse arrest in $1(4.54 \%)$ patient.

Majority of neonates, $110(78.57 \%)$ in all patients had APGAR score $>7$ at $1 \mathrm{~min}$ and 5 min after birth. $4(2.85 \%)$ babies of vaginally delivered babies with curve in right of alert line were admitted for observation in NICU for respiratory distress. $12(8.57 \%)$ neonate of patients who crossed alert line but delivered vaginally (including 3 vaccum deliveries) had APGAR $<7$ but were shifted out of NICU within 48 hours. 10 (7.14\%) neonates of patients delivered by LSCS had APGAR $<7$ at 1minute, 5 were in NICU for 6 hours then shifted mother side and 4 were in NICU for 3-7 days and then discharged. 1 neonate of LSCS patient with polyhydramnios had trachea-oesophageal fistula (TOF)diagnosed at birth, operated and is now mother side (table $3 \& 4)$.

There were 4 babies, who developed neonatal jaundice on $4^{\text {th }}$ postpartum day and managed conservatively. There were no perinatal deaths.

Maternal complications were seen in $3.57 \%$ of patients namely atonic PPH (2), extended episiotomy (2) and precipitate labour with complete perineal tear (1) were seen in cases of abnormal progress of labour, but due to high vigilance and preparedness due to continuous graph, complications were fore seen and managed immediately (table 4). There were no cases of traumatic PPH, bladder injury, puerperal pyrexia or peripartum hysterectomy and maternal death.

Table 1: General characteristics of study participants

\begin{tabular}{|l|c|}
\hline Variables & \\
\hline Age (years) & $23.13 \pm 1.08$ \\
\hline BMI & $22.77 \pm 0.97$ \\
\hline GA (Weeks) & $39.30 \pm 1.01$ \\
\hline Cervical Dilation & $4.41 \pm 0.63$ \\
\hline
\end{tabular}

Table 2: Mode of delivery

\begin{tabular}{|l|c|}
\hline Mode of delivery & Number of subjects \\
\hline Vaginal delivery & $118(84.28 \%)$ \\
\hline Caesarean Section & $22(15.71 \%)$ \\
\hline Total & $140(100 \%)$ \\
\hline
\end{tabular}

Table 3: Apgar score of Neonates

\begin{tabular}{|l|c|c|}
\hline Parameters & No. & Percentage \\
\hline Apgar Score $>7$ at 1 min. \& 5 min. & 110 & $78.57 \%$ \\
\hline Apgar Score $<7$ at 1 min. \& 5 min. & 30 & $21.42 \%$ \\
\hline
\end{tabular}

Table 4: Neonatal outcome

\begin{tabular}{|l|c|c|}
\hline Parameters & No. & Percentage \\
\hline Post partum neonatal jaundice & 4 & $2.85 \%$ \\
\hline Respiratory distress & 4 & $2.85 \%$ \\
\hline Anomaly-TEF & 1 & $0.71 \%$ \\
\hline
\end{tabular}

Table 5: Maternal outcome $(\mathrm{N}=5 / 140)$

\begin{tabular}{|l|c|}
\hline Maternal Complications & Number of subjects \\
\hline Complete perinatal tear & $1(0.71 \%)$ \\
\hline PPH & $2(1.42 \%)$ \\
\hline extended episiotomy & $2(1.42 \%)$ \\
\hline
\end{tabular}




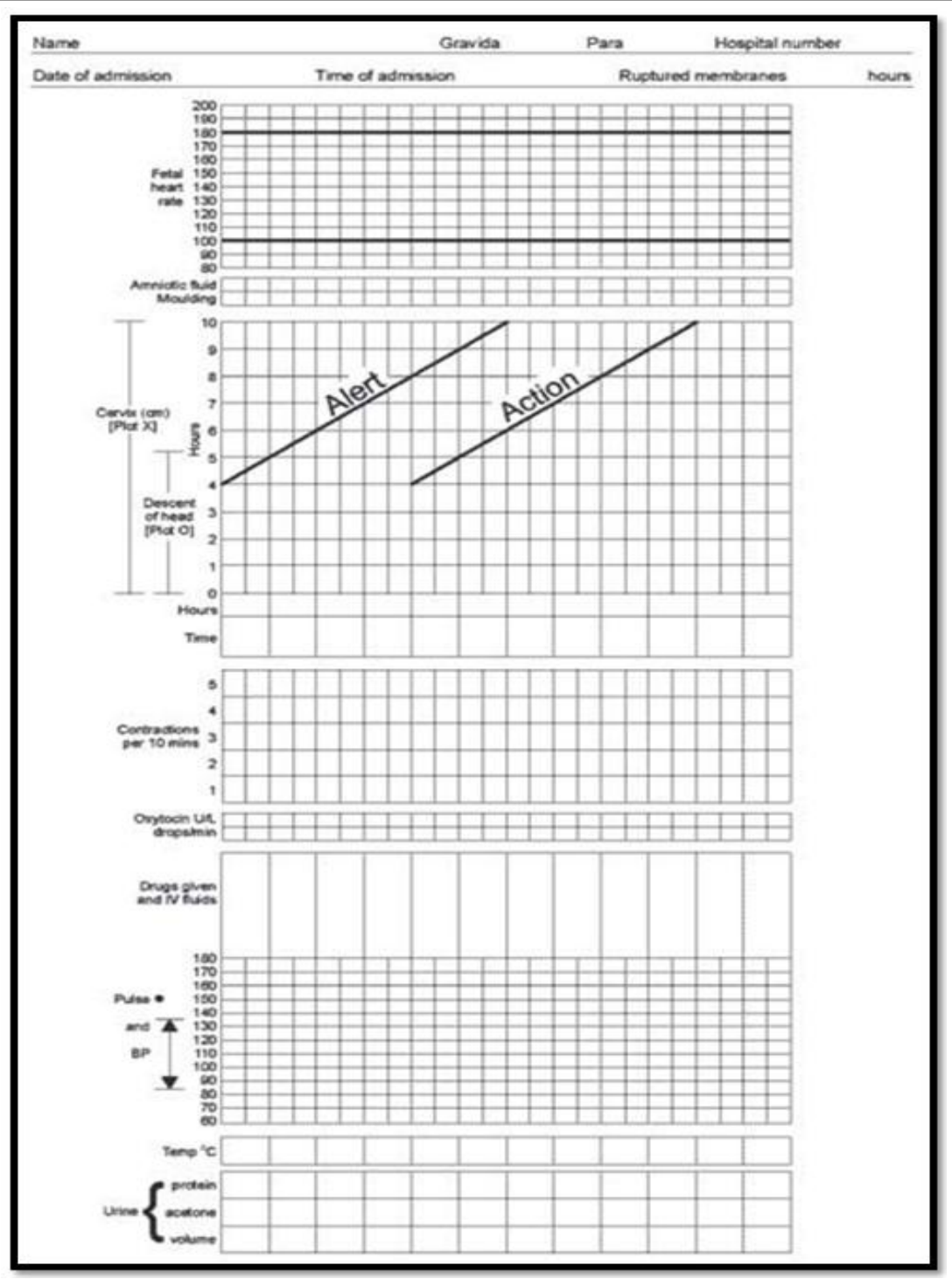

Figure 1: Modified WHO Partograph

\section{Discussion}

WHO modified partograph is a pre-printed single sheet inexpensive method of labour monitoring. Though use is widely recommended, it is still not been used properly. ${ }^{7}$

In our study, 140 nulliparous patients in active labour were monitored using WHO modified partograph and it's effect on maternal and neonatal outcomes was analyzed.

In our study, age and BMI had no relation with partograph pattern. Majority of patient had vaginal delivery as seen in other studies of Sethi et $\mathrm{al}^{8}$, Lakshmidevi et $\mathrm{al}^{9}$. Delayed progress of labour was mainly indicated by the duration of the active phase of labour and shift of curve towards right of alert line. Timely intervention in case of slow progress in form of reasessement, artificial rupture of membrane, oxytocin etc resulted in successful vaginal deliveries. With shifting of curve to right of alert or action line, chances of instrumental deliveries and LSCS increased as seen in studies of Lakshmidevi et $\mathrm{al}^{9}(2012)$. The results are comparable to the studies by Impey et $\mathrm{al}^{10}$ (2000). Though there is an increasing trend of LSCS as compared to instrumental deliveries especially forceps, in our study for prolonged second stage, vaccum delivery was preferred.

In a study of 72,593 mothers from Sweden ${ }^{11}$ (2008-2012), it was confirmed that risk of maternal postpartum complications like infectious disease, urinary retention, traumatic $\mathrm{PPH}$, fistula etc increase with increasing duration of active labour especially second stage proper monitoring by partograph decreases these complications to a 
great extent. There were no such complications in our study. Complications like mild atonic PPH in 2 patients was probably idiopathic though both patients had labour curve between alert and action line and needed oxytocin augmentation. Both cases were managed successfully by medical management. One patient who had precipitate labour that is combined duration of $1^{\text {st }}$ and $2^{\text {nd }}$ stage of labour under 2 hours, had unusually strong uterine contraction and had baby weight of $3.8 \mathrm{~kg}$, though complete perineal tear occurred, which was repaired immediately and successfully. In our study, most common indication of cesearean section was non progress of labour $(15.71 \%)$, followed by fetal distress and meconium stained liquor in first stage of labour (2.85\%). In recent Indian study at Punjab, main indication of LSCS was fetal distress in 27/200 patients followed by non progress of labour in only $5 / 200$ patients.

There was no neonatal or maternal mortality in our study, which signifies the inverse relationship between proper monitoring and complication rate. In a study by Tayade and Jadho $(2012)^{12}$ at Wardha, comparing results between no partograph and modified WHO partograph, it was seen that neonatal and maternal morbidity reduced significantly with use of partograph. In a GermanTurkish study by Orji et al. (2007) $)^{13}$, there was one case of uterine rupture and two maternal deaths before partograph use but none after use of partograph.

Most babies (78.57\%) had spontaneous cry and APGAR $\geq 7$ at 1 and 5 minutes and there was no neonatal mortality. There were more NICU admissions if labour curve shifts beyond alert line (18.57\%). This shows that with abnormal progress of labour, neonatal distress may increase. In a similar study by Penamadu et al.2014 ${ }^{14}, 92.4 \%$ of neonates had APGAR $\geq 7$ at 1 and 5 minutes. Though NICU care was required in $8 \%$ of babies, they were shifted to mother side within 4 days. So, by proper partographic monitoring, more vigilance can be offered to babies of mother crossing alert line though every neonates needs pediatric evaluation at birth. All anomalies also cannot be diagnosed in antenatal period like trachea oesophageal fistula case in our study. Postnatal detailed evaluation is must. Partograph may also serve as a medico- legal document, stamping proper monitoring by health care personnel in event of any untoward sequence or medico legal litigation.

\section{Conclusion}

With our study, we concluded that modified WHO partograph is a simple, inexpensive and reliable method to provide a continuous pictorial depiction of events of labour. It helps in early identification of abnormal labour patterns, timely intervention and augmention, if required. After simple training, it can be easily used by midwives, nurses and health personnel in primary health care centre. If this graph is attached with referral slip to higher centre, it gives a good overall idea of labour events and treatment given. It improves labour outcome and decrease intrapartum and postpartum complication rate.

Though it's introduction way back and literature confirming it's benefits, it is still not used universally to its full extent even in developing countries. We suggest that in a country like India, where still maternal morbidity and mortality rate is high, partograph use should be implemented in all hospitals and labour rooms for better obstetric care and attainment of Millennium Development Goal of improving maternal health.

Funding - no funding sources

Conflict of interest - none declared.

\section{References}

1. Friedman EA. Primigravid labour. A graphicostatistical analysis. Obstetrics and Gynecology, 1955, 6(6):567-589.

2. Philpott RH, Castle WM. Cervicographs in the management of labour in primigravidae. Journal of Obstetrics and Gynaecology of the British Common wealth. 1972;79:599-602. 
3. Royal College of Obstetricians and Gynaecologists. Induction of labour. London RCOG Press 1998, Guidance No 16.

4. WHO 1994, World Health Organization partograph in management of labour World Health Organization Maternal Health and Safe Motherhood Programme. Lancet.1994;343(8910):1399-404.

5. World health statistics 2016, www.who.int.healthinfo.statistics.in www.nitiaayog.gov.in

6. Montgomery, Ann L. et al. "Maternal Mortality in India: Causes and Healthcare Service Use Based on a Nationally Representative Survey." Ed. Zulfiqar A. Bhutta. PLoS ONE $9.1 \quad$ (2014): e83331. PMC. Web. 22 June 2018.

7. Yisma E, Dessalegn B, Astatkie A, Fesseha N. Completion of the modified World Health Organization (WHO) partograph during labour in public health institutions of Addis Ababa, Ethiopia. Reproductive Health. 2013;10:23. doi:10.1186/1742-4755-10-23.

8. Prachi Sarin Sethi, Sujata Sharma, Indu Chawla. Comparative evaluation of composite and simplified who partograms in a tertiary care centre in North India. Int J Reprod Contracept Obstet Gynecol. 2017 Nov;6(11):5003-5007.

9. Lakshmidevi M, Malini KV, Shetty VH. Partographic Analysis of Spontaneous Labour at Term in Primigravida. Journal of Obstetrics and Gynaecology of India. 2012;62(6):635-640.

10. Impey L, Hobson J, O’ Herlihy C. Graphic analysis of actively managed labor: prospective computation of labour progress in 500 consecutive nulliparous women in spontaneous labor at term. Am J Obstet Gynecol. 2000;183(2):438-443.
11. Stephansson O, Sandstrom A, Petersson G, Wikstrom A-K, Cnattingius S. Prolonged second stage of labour, maternal infectious disease, urinary retention and other the early complications in postpartum period. BJOG 2016;123:608-616.

12. Tayade, Surekha; Jadhao, Pooja. The Impact of use of Modified who Partograph on Maternal and Perinatal Outcome. International Journal of Biomedical and Advance Research, [S.1.], v. 3, n. 4, p. 256262, may 2012. ISSN 2229-3809.

13. Orji EO, Adeyemi BA, Makinde NO, Fatusi AA, Onwudiegwu U. Impact of training on the use of partograph on maternal and perinatal outcome in peripheral health centers. J Turk Ger Gynecol Assoc 2007;8:148-154.

14. Penumadu KM, Hariharan C. Role of partogram in the management of spontaneous labour in primigravida and multigravida. Int $\mathbf{J}$ Reprod Contracept Obstet Gynecol 2014;3:1043-9. 